\title{
Aportes para las representaciones sobre la sexualidad en la disponibilidad léxica de estudiantes secundarios de la región NEA*
}

\author{
Natalia Virginia Colombo \\ Olga Natalia Trevisán \\ Hugo Roberto Wingeyer*
}

\section{Resumen}

En este trabajo aportamos datos para el estudio de las representaciones sociales sobre la sexualidad a partir de la identificación y análisis del léxico sincrónico disponible en jóvenes de la región NEA de Argentina. El cálculo de la disponibilidad léxica, para el que utilizamos el programa LEXIDISP, patrocinado por el Instituto Cervantes, la Universidad de Alcalá y la Asociación de Lingüística y Filología de América Latina, se basa en la aplicación de pruebas

\footnotetext{
* Artículo recibido el 1 de junio de 2013. Aceptado el 25 de octubre de 2013.

* Natalia Virginia Colombo es Doctora en Semiótica, Centro de Estudios Avanzados y Facultad de Filosofía y Humanidades de la Universidad Nacional de Córdoba. Profesora Adjunta, a cargo y por concurso, de Semiótica del Departamento de Letras de la Facultad de Humanidades de la UNNE.

Contacto: nvcolombo@gmail.com
}

Olga Natalia Trevisán es Profesora en en Letras, Facultad de Humanidades, Universidad Nacional del Nordeste. Profesora Adjunta, a cargo y por concurso, en Lengua y Cultura Latinas, del Departamento de Letras de la Facultad de Humanidades de la UNNE.

Contacto: ontrevisan@gmail.com

Hugo Roberto Wingeyer es Doctor por la Universidad de Alcalá, en el Programa en Lingüística Aplicada. Profesor Titular de Historia del Español, por concurso, del Departamento de Letras de la Facultad de Humanidades de la UNNE

Contacto: hugowingeyer@hotmail.com 
asociativas determinadas por temas o centros de interés. Para ser más precisos, seleccionamos aquí temas en los que aparecen unidades que refieren a la sexualidad: Partes del cuerpo, Profesiones y oficios, Juegos y distracciones y ¿Cómo insultamos? Las representaciones se basan en las relaciones entre las manifestaciones sincrónicas concretas del lenguaje de las ciudades de Resistencia y Corrientes, tomadas en conjuntos estratégicos: las partes del cuerpo relacionadas con el sexo, la prostitución, la masturbación, enfermedades de transmisión sexual, el coito, diversidad sexual: homosexualidad, transexualidad y bisexualidad, y la mujer histérica.

\section{Palabras clave}

Representación social - léxico disponible - sexualidad.

\section{Abstract}

In this paper we provide data for the study of social representations on sexuality, based on the identification and analysis of young people's synchronic lexicon available, in the northeast region of Argentina. The estimation of lexical availability, for which we used the LEXIDISP software sponsored by the Cervantes Institute, the University of Alcalá and the Latin American Association of Linguistics and Philology, was based on the application of association tests organized by topics or centers of interest. To be more precise, we selected topics which generate lexical units related to sexuality: Parts of the Body, Professions and Occupations, Games and entertainment and How do we insult?

The representations are based on the relations of concrete synchronic language productions sampled in the cities of Resistencia and Corrientes and based on strategic categories: parts of the body related to sex, prostitution, sexual intercourse, masturbation, sexually transmitted diseases, sexual diversity: homosexuality, transsexuality, bisexuality and hysterical women.

\section{Keywords}

Social representation - lexical availabity - sexuality. 


\section{Consideraciones teórico-metodológicas}

Nos proponemos en este trabajo aportar información para el estudio de las representaciones sociales de la sexualidad a partir del análisis de los sentidos posibles de vocablos que la refieren en dos de las cuatro capitales de la región NEA de Argentina, Resistencia (2001) y Corrientes (2008).

En primer lugar, para los aspectos relacionados con las representaciones sociales, tomamos a Jodelet (1985, pp. 470-479), quien explica que hay diversas ópticas en la formulación de cómo se elabora la construcción psicológica y social de una representación social. Consideramos pertinente aquí la perspectiva que trata la representación como una forma de discurso, que desprende sus características de las prácticas discursivas de sujetos situados en la sociedad. La información puede obtenerse, como en el caso de la disponibilidad léxica, en situaciones experimentales, con datos recopilados mediante procedimientos pautados.

Nos valemos, por lo tanto, del léxico sincrónico disponible de 1001 estudiantes secundarios de los primeros y de los últimos cursos. Los resultados de la disponibilidad léxica se basan en la aplicación de pruebas asociativas, determinadas por veinticuatro temas, centros de interés o campos semánticos con los que se intenta cubrir el mayor número de intereses humanos. Además de los que abordamos en este trabajo: Partes del cuerpo, ¿Cómo insultamos?, Profesiones y oficios y Juegos y distracciones, seleccionados sobre la base de la presencia de unidades que refieren a la sexualidad, se toman en consideración diferentes temas, como La ropa, Partes de la casa (sin los muebles), Los muebles de la casa, Alimentos y bebidas, Objetos colocados en la mesa para la comida, La cocina y sus utensilios, La escuela: muebles y materiales, Iluminación, calefacción y medios para airear o refrescar un ambiente, La ciudad, Mitos populares, El sida, Los animales y Los colores, entre otros no menos importantes. Observamos que es a partir de la motivación del nombre de cada uno de los centros de interés que el informante, en un tiempo de dos minutos, anota todos los vocablos que asocia con el centro en cuestión, tanto los términos que usa como los que conoce.

Para el cálculo del índice de disponibilidad, que combina la frecuencia con el orden de aparición de las unidades, utilizamos el programa estadístico LEXIDISP, avalado por el Instituto Cervantes, la Universidad de Alcalá y la Asociación de Lingüística y Filología de América Latina. Dicho programa trabaja con palabras, que son todos los términos recogidos, y con vocablos, que son cada una de las entradas diferentes.

En cuanto a los factores sociales, tomamos en cuenta en la metodología de la disponibilidad léxica las siguientes variables: sexo, escuela del centro y de la periferia, escuela pública y privada, nivel socioeconómico: alto, medio y bajo, y edad de los 
informantes. Observamos que en este trabajo nos centramos en las variables sexo y tipo de escuela: pública y privada.

Para el análisis propiamente dicho consideramos relevante tratar los límites de los trabajos basados en los resultados de la disponibilidad en relación con metodologías que operan con un léxico contextual, obtenido en actuaciones reales. Nos referimos a lo que Mateo García llama "neutralización de los factores pragmático-discursivos", ya que se trabaja con listas de unidades "elaboradas por test" (1996, p. 52).

Ahora bien, creemos que es posible relativizar los límites de esta propuesta en lo que se refiere a la obtención de datos, si consideramos que la disponibilidad es "el caudal léxico utilizable en una situación comunicativa dada", recogido "a partir de unos estímulos verbales", que son los centros de interés (López Morales, 1999, p. 11).

En cuanto al ámbito de la investigación, se trata de la región NEA, acorde con la división del español de Argentina que propone Donni de Mirande (1992, pp. 384-400), determinada por diversos factores: situación geográfica, formación histórica, evolución demográfica, aspectos culturales y condiciones socioeconómicas. Se caracteriza, además de la lógica presencia de rasgos comunes a otras áreas hispánicas, por la retención de modalidades tradicionales y por la presencia de lenguas indígenas.

Otra cuestión relevante, relacionada con el ámbito de la investigación, es la necesidad de considerar los resultados como un todo, como una unidad de partes que se relacionan; para eso, acorde con Lotman (1996, pp. 26-27), pensamos la región NEA como una semiosfera, en la que su frontera "se intersecta con las fronteras de los espacios culturales particulares"; todavía más, cuando, como en este caso, el "espacio cultural tiene un carácter territorial, la frontera adquiere un sentido espacial en el significado elemental". Dentro de este gran espacio cultural, analizamos, a su vez, los resultados de la disponibilidad léxica de Resistencia y de Corrientes, ciudades muy próximas entre sí, como una zona en la que se entrecruzan múltiples espacios culturales particulares.

En la misma línea, los temas o centros de interés componen también un conjunto de conjuntos con intersecciones, con rasgos exclusivos propios de cada tema, de acuerdo con el perfil sociocultural de su motivación, pero a la vez con elementos, en este caso vocablos, compartidos con otros.

En lo que respecta, entonces, a los centros de interés abordados, consideramos, en primera instancia, el grado de coincidencia de las respuestas obtenidas en las encuestas; para eso, aplicamos el índice de cohesión. Este índice (con escala del 1 al 0), que se obtiene dividiendo el promedio de respuestas de cada sujeto por el número de palabras diferentes, será menor cuando mayor variación exista en las respuestas. Informa así si el centro analizado es cerrado o difuso (Echeverría, 1987; 1991).

Clasificamos, entonces, al centro Partes del cuerpo entre los más cerrados, porque el conjunto del léxico que describe el cuerpo humano, que favorece además la aparición de nombres concretos, es compartido por la mayor parte de los informantes. En tanto 
que los centros Profesiones y oficios, en el que los informantes responden, básicamente, a la pregunta: ¿de qué vive la gente?, y Juegos y distracciones, en el que mencionan distintas formas de entretenimiento, son mucho más abiertos o difusos que el anterior.

Por último, vale la pena detenernos en el tema ¿Cómo insultamos?, porque se encuentra entre los más abiertos y complejos debido a su motivación: la necesidad, por parte del informante, de tener que imaginarse la presencia de otra persona en el momento del registro de datos. Partimos para la precisión de su perfil de la definición del término insultar, propuesta por el DRAE (1992): "Ofender a uno provocándolo e irritándolo con palabras o acciones". Nos encontramos, en primera instancia, ante un acto de habla que implica atribuir a otros comportamientos considerados reprobables. El insulto arquetípico es, entonces, la asignación, por parte del hablante, de una calificación negativa a un destinatario sobre una práctica, conducta o cualidad.

Los insultos, incluidos en los actos de habla comportativos, "constituyen un grupo muy heterogéneo, y tienen que ver con las actitudes y con el comportamiento social; por ejemplo, pedir disculpas, felicitar, elogiar, dar pésame, maldecir y desafiar" (Austin, 2003, p. 199) y, por otro lado, "incluyen la idea de reacción frente a la conducta y fortuna de los demás, y las actitudes y expresiones de actitudes frente a la conducta pasada o inminente del prójimo" (Austin, 2003, p. 207).

Observamos, finalmente, que todos los centros se estructuran sobre la base de dos espacios: el núcleo, compacto o cerrado, conformado por los veinte primeros vocablos más disponibles, y la periferia, que va adquiriendo un carácter más difuso o abierto a medida que los términos que la componen se alejan de las unidades que conforman el núcleo.

Ahora bien, en lo que respecta a los vocablos seleccionados en los cuatro centros de interés, los presentamos no como un simple repertorio, sino como un conjunto de unidades que puede dar cuenta de las representaciones sociales de la sexualidad en esta comunidad. Para eso, en principio, nos es útil la diversidad de aspectos -físicos, psicológicos y sociales - presentes en los dos conceptos de sexualidad que propone el DRAE (2001), conceptos que son de gran utilidad a la hora de sistematizar la información necesaria para la elaboración de sus representaciones. La sexualidad implica, por un lado, un "conjunto de condiciones anatómicas y fisiológicas que caracteriza a cada sexo"; y por otro, hábitos y conductas relacionadas con el "apetito sexual, la propensión al placer carnal". Si vinculamos ambas dimensiones con los centros de interés analizados, observamos que la primera se ajusta a la información obtenida en el centro Partes del cuerpo; mientras que la segunda, en la que subyacen aspectos conductuales y sociales, concuerda, por sus perfiles, con la información de los centros Profesiones y oficios, ¿Cómo insultamos? y Juegos y distracciones.

Para ampliar y precisar el marco teórico referido a la sexualidad, nos valemos del concepto de "dispositivo de sexualidad" de Foucault (1985, p. 128), entendido como un 
conjunto decididamente heterogéneo, que comprende discursos, instituciones, instalaciones arquitectónicas, medidas administrativas, enunciados científicos, proposiciones filosóficas, morales, filantrópicas; en resumen, los elementos del dispositivo pertenecen tanto a lo dicho como a lo no dicho. El dispositivo es la red que puede establecerse entre estos elementos.

Aclaramos que en esta propuesta nos centramos en un aspecto del "dispositivo", las relaciones que podamos establecer en las manifestaciones sincrónicas concretas del lenguaje de las ciudades de Resistencia y Corrientes, tomadas en conjuntos estratégicos: las partes del cuerpo relacionadas con el sexo, la prostitución, la masturbación, enfermedades de transmisión sexual, el coito, diversidad sexual homosexualidad, transexualidad y bisexualidad- y la mujer histérica. Conjuntos estratégicos que, en términos de Foucault (2011, p. 100), "despliegan a propósito del sexo dispositivos específicos de saber y de poder".

Conviene insistir, en este punto, en que tomamos solo un aspecto del "dispositivo" de Foucault porque este trabajo se basa en los resultados de la metodología de la disponibilidad léxica: listados de términos obtenidos de pruebas asociativas. Conviene insistir también en la relativización de los límites de la disponibilidad: los nombres de los diferentes centros de interés como motivadores de situaciones de comunicación, en el momento en el que se aplican las encuestas.

Vamos ahora a nuestro punto de partida para su descripción, los siguientes interrogantes: ¿de qué hablan actualmente los jóvenes del NEA cuando hablan de sexualidad? y ¿qué representaciones sociales se pueden construir con ese contenido? En este sentido, retomamos a Jodelet (1985, p. 473), quien entiende por representación social una manera de interpretar y de pensar una realidad cotidiana, una forma de conocimiento social, construido a partir de experiencias, informaciones, conocimientos y modelos de pensamientos que se reciben y transmiten a través de la tradición, la educación y la comunicación social. Este conocimiento es, en muchos aspectos, socialmente elaborado y compartido; un saber práctico que participa en la construcción social de una realidad común a un conjunto social e intenta dominar esencialmente ese entorno, comprender y explicar los hechos e ideas de ese contexto. Por consiguiente, es, al mismo tiempo, un producto y un proceso de una actividad de apropiación de una realidad externa y de elaboración psicológica y social de esa realidad.

En síntesis, las representaciones sobre la sexualidad, elaboradas a partir de los resultados de determinados centros de interés del léxico sincrónico disponible de los jóvenes del NEA, no solo expresan lo que esos sujetos piensan o interpretan, sino también contribuyen a constituirlas y son referencias o modos de comprender la realidad. 


\section{Análisis de unidades que, en los diferentes centros de interés, refieren a la sexualidad}

En esta instancia del análisis nos detenemos tanto en aspectos cuantitativos como cualitativos de los resultados de la disponibilidad léxica en cada uno de los centros considerados en este trabajo, acorde con sus diferentes motivaciones.

\section{Partes del cuerpo}

Este centro, en el que nos encontramos con una mayoritaria presencia de voces del español general de léxico estandarizado y varios coloquialismos, es el de menor complejidad asociativa debido a su delimitación semántica.

Para su análisis, tomamos en cuenta las consideraciones realizadas en un trabajo previo (Wingeyer, Gusberti y Trevisán, 2011), en el que se trató el tema de las partes del cuerpo en los resultados de la disponibilidad léxica de Resistencia y Corrientes, desde otras perspectivas.

En primer lugar, en lo que respecta a información cuantitativa sobre partes del cuerpo relacionadas con el sexo, en la primera recogida de datos, en 2001, fueron encuestadas 281 mujeres, de las cuales 148 se refirieron a las anatomías masculina y femenina (el $53 \%$ ) y 220 hombres, de los cuales 134 mencionaron el tema (el $61 \%$ ); en el segundo sondeo en 2008, fueron encuestadas 320 mujeres, de las cuales 232 mencionaron partes del cuerpo relacionadas con el sexo - lo que representa el 73 \%y 180 varones, de los cuales 148 se refirieron al tema (el $82 \%$ ).

Observamos que aunque el número de informantes mujeres es mayor en ambas muestras, el número de varones que alude a las anatomías masculina y femenina es superior. Notamos además que se registra un crecimiento paralelo entre encuestados de ambos sexos en las muestras 2001-2008, ya que el porcentaje de mujeres se eleva del 53 al 73 \% y en los varones, el aumento es de 61 a $82 \%$; en ambos casos se trata de un incremento del $20 \%$.

En la primera muestra, solo el $56 \%$ de los encuestados menciona partes de su cuerpo asociadas con la genitalidad. En la segunda, notamos un incremento significativo del $76 \%$. Este aumento se registra no solo en el número de encuestados sino también en la cantidad de palabras y en la variedad de vocablos; así, en relación con ambas anatomías, en la primera muestra leemos 523 palabras sobre un total de 9.173, lo que representa el 5,7\%, mientras que en la segunda, observamos 1.163 sobre un total de 11.305, es decir, el 10,3\%. Si nos referimos a vocablos, en 2001 se distinguen 42 y más del doble (89) en 2008, sosteniendo esta tendencia.

En segundo lugar, en cuanto a aspectos cualitativos, de los datos se desprende que los varones aluden con mayor libertad a las partes del cuerpo relacionadas con el sexo 
en ambas muestras, y que el número de vocablos para nombrar ambas anatomías es semejante. Se registraron 32 vocablos para referir la femenina y 34 para la masculina.

La muestra revela, en 2001, una escasa presencia de términos concretos relacionados con la sexualidad en relación con la cantidad de palabras registradas. Los vocablos que aparecen son significativos, ya que corresponden, en general, a términos técnicos: vagina, clítoris, ovario, óvulo, útero, trompa de Falopio, monte de Venus, mama, seno, pene, testículo, espermatozoide y próstata, y a coloquialismos: concha, teta, pito, chilo, pija y bolas. Es notable el predominio de los primeros.

Por el contrario, en la muestra 2008, hallamos un incremento y variedad de los vocablos que designan los órganos sexuales por su forma anatómica (empanada, batata, manguera), que aluden a objetos puntiagudos y afilados en el caso del órgano sexual masculino (verga) o de hendiduras para el femenino (hachazo), otros que aluden a su funcionalidad procreadora (manguera de semen); también eufemismos a través de nombres de animales (papirola, nutria).

Encontramos interesante la descripción de las diferencias de género de los informantes en relación con lo expuesto. Así, en la muestra 2001, las mujeres se refieren a su genitalidad con 11 vocablos: vagina, clítoris, ovario, óvulo, trompa de falopio, concha, monte de venus, mama, seno, teta. En 2008, otra vez las mujeres, registran un caudal léxico superior al anterior, con 25 vocablos en total. Se agregan a los ya mencionados vulva, cachí, cajeta, hachazo, la amiga, pachula, papo, papona, papu, pochola, glándula mamaria, goma, lila y lola, lola, pezón.

Cobran relevancia en esta muestra los eufemismos (lila y lola, la amiga, pachula, papu, papona, pachola, cachi) y los disfemismos (hachazo, cajeta, papo), aun cuando persiste la preferencia por los tecnicismos en la cantidad de palabras registradas. Notamos que se incorporan 9 vocablos con el significado de vagina: 3 eufemismos y 6 disfemismos. La forma coloquial concha registra un aumento significativo en 2008: de frecuencia 3 pasa a 28; lo mismo sucede con teta: de 6 menciones pasa a 24, y con trompa de Falopio, que pasa de 3 a 24. Esto revela un incremento de los coloquialismos y de los tecnicismos ya mencionados.

En cuanto a los vocablos referidos a la sexualidad masculina, en 2001 las mujeres registran solo 5: pene, testículo, bolas, espermatozoide y próstata. En 2008 incorporan los siguientes coloquialismos: picho, pito, chilo, pija, huevo, los eufemismos pilín, pitilín, pirulín, pichilo, el amigo, los disfemismos batata, chota, poronga, pinga y los tecnicismos escroto, epidídimo, esperma, glande y gónada. A pesar de la variedad, el total de palabras para referirse a pene es de 68 , frente a 98 . Es decir, nuevamente, la variedad no va en detrimento del uso de términos técnicos.

En el análisis de términos usados por los varones, distinguimos en 200112 vocablos para referirse a la sexualidad femenina. No registran allí mama (usado únicamente por las mujeres), e incorporan pezón y goma (que las mujeres no registran en esa muestra). 
En 2008, incorporan 27 vocablos; agregan a los utilizados por las mujeres: cachula, cachuncha, cachunfleta, empanada, papula y tetona.

Es significativa la frecuencia de disfemismos en los varones (hachazo, empanada y papo son ejemplos de ello, puesto que suman 24 palabras entre varones, frente a 2 entre mujeres). Es también menor la frecuencia de términos técnicos o especializados en estos informantes: frente a 114 registros de vagina en mujeres, 59 en varones; 45 de útero frente a 7; 56 de ovario frente a $11 ; 29$ de seno frente a 12.

Respecto de su propia sexualidad, en 2001 registramos 10 vocablos: pene, picho, chilo, pija, pito, testículo, huevo, esperma, espermatozoide y prepucio. En 2008 se agregan: verga, pilín, pitilín, papirola, chota, choto, poronga, falo, nutria, manguera de semen, polla, chingada, huevo, escroto, eyaculación, semen y próstata.

El uso de términos técnicos no registra un aumento significativo entre los varones; por el contrario, se mantiene constante, aun con tamaña variedad de vocablos: en 2001 registramos frecuencia 99 en pene frente a 101 en 2008; 30 en testículo frente a 35 y 3 en espermatozoide, frente a 1. Es llamativo el aumento de palabras para referirse a pene; así se registran 69 frente a las 101 mencionadas. En otras palabras, los varones registran 12 nuevos vocablos con el significado de pene.

Destacamos la incorporación de tecnicismos que aparecen por primera vez en 2008 : escroto y vulva (en ambos sexos); ovario, glándula mamaria, epidídimo, gónada y glande, en mujeres. $\mathrm{Y}$, significativamente, mama, espermatozoide y los fluidos menstruación, eyaculación y semen, solo en varones. Por otra parte, nos llama la atención la desaparición de óvulo y prepucio entre los varones, y de espermatozoide y monte de Venus entre las mujeres, en la misma muestra; también la incorporación de pezón y esperma entre mujeres, y mama entre varones, ya existentes en el sexo opuesto en la muestra 2001.

A propósito de excepciones, solo en mujeres aparecen los siguientes coloquialismos: garcha, batata, pinga, bolas (llamativamente, tanto en 2001 como en 2008) y huevo (en 2008), y son exclusivos en varones: papirola, choto, poronga, falo, nutria, manguera de semen, polla, chingada.

Surgen abundantes eufemismos que, suponemos, son usados en contextos familiares o propios de la relación adulto-niño en relación con esta temática: pilín, pitilín, pirulín, pichilo, pachula, papula, papona, papu, pachola, cachi, cachula, cachuncha y cachunlfleta. La frecuencia de uso de estos términos se da, para órgano masculino, 4 vocablos en mujeres (en total 8 palabras) y 2 vocablos en varones (en total 6 palabras); mientras que para órgano femenino, 5 vocablos en mujeres (en total 10 palabras) y 7 vocablos en varones (en total 22 palabras).

En lo concerniente a la variable clase social destacamos que en la muestra 2001 no hay diferencias relevantes en el uso de términos en las tres clases sociales consideradas. Ciertamente esto se modifica en la muestra 2008: el mayor número de disfemismos se registra en la clase alta, en tanto que la media y la baja priorizan el uso de 
coloquialismos y tecnicismos. A modo de ejemplo, el único registro de los vocablos gónada y epidídimo se da en mujeres de clase baja. En síntesis, exceptuando lo antedicho, la lectura cualitativa de datos según esta variable no nos resulta tan significativa como la relacionada con la variable sexo.

Por último, nos interesa mencionar también términos referidos a fluidos, como menstruación, semen y eyaculación -empleados solo por varones-, y otro término indirectamente relacionado con el tema del centro, el producto de la concepción: embrión.

\section{Profesiones y oficios}

Si bien este centro tiene una motivación muy concreta, el carácter mucho más abierto en relación con Partes del cuerpo se debe a que, además de las profesiones y oficios tradicionales y reconocidos, nuestros informantes han incluido otras actividades menos convencionales.

En primer lugar, consideramos términos como prostíbulo, prostitución, prostituto, taxi boy, stripper, striptease, travesti, trolo, puto y puta que designen prácticas sexuales dispersas, algunas de las cuales podrían asociarse, directa o indirectamente, a la prostitución; por ende, en este centro se estarían poniendo en evidencia problemáticas sociales vinculadas con la exclusión social y la falta de trabajo, incluso la esclavitud sexual y la trata de personas, entre otras cuestiones.

En segundo lugar, un comentario especial merece la práctica sexual que subyace en el término violador, que, al ser considerada como profesión, aparecería como una curiosa naturalización en el imaginario de los jóvenes de nuestra sociedad. Observamos que este acceso carnal, que menoscaba la dignidad humana y atenta contra el derecho a la libertad sexual, es mencionado mayoritariamente por mujeres, quienes, de cierta manera, estarían denunciando y poniendo en evidencia conductas sexuales violentas.

\section{Juegos y distracciones}

En este centro, que ofrece, desde el punto de vista de la naturaleza de las asociaciones, muchas más posibilidades que Profesiones y Oficios, la presencia de términos sobre la sexualidad es significativa.

Registramos tres vocablos referidos a uno de los conjuntos estratégicos del dispositivo de sexualidad, la masturbación: pajearse, pajeros, hacerse la paja, y nueve relacionados con el coito: teto, tu zanjón y mi tripita, culear, coger, joder, la mamá y el papá, orgía, la basurita, hacer el amor — solo en mujeres-y la tocadita.

Desde el punto de vista de la motivación de este centro de interés, el registro de términos que se vinculan con juegos y con distracciones, y determinados lexemas podrían recibir los calificativos de falocráticos y violentos: joder, coger, hacerse la paja, pajeros, culear. Sin embargo, otra es la calificación posible de las unidades: la mamá y 
el papá y hacer el amor —usados casi exclusivamente por mujeres-, con innegables connotaciones afectivas.

Por último, sobre la base de su aparición solo en informantes hombres, la unidad venus, en referencia al canal de televisión por cable, cuya programación se basa en contenido erótico, podría ser considerada como una distracción exclusivamente masculina.

\section{¿Cómo insultamos?}

Nos interesa conocer en este centro -propuesto al grupo correntino de informantes en 2008, cuya propia formulación hace que sea el más difuso de los cuatro- cuáles son las cualidades o conductas que los jóvenes estiman con respecto a la sexualidad, para poder formular hipótesis respecto de los valores sociales convenidos en la región NEA sobre este tema.

A pesar de la gran variedad en la naturaleza de las asociaciones y en las unidades recogidas, cuando se clasifican los vocablos, las esferas a las que aluden se acotan considerablemente. De un registro de 545 vocablos, 242 se refieren a la sexualidad. En otras palabras, el mayor porcentaje de insultos (44.4\%) se centra en la conducta sexual, tanto de varones como de mujeres; le siguen en orden de importancia el funcionamiento de la inteligencia, la salud mental o la salud en general, las conductas socialmente reprobadas $y$, finalmente, insultos referidos al aspecto físico.

Como ya mencionamos en el punto anterior, en esta muestra 320 mujeres y 180 varones fueron encuestados. Entre las primeras, registramos 2282 palabras y 1540 entre los segundos, es decir que, sobre un total de 3822 términos, corresponde a las mujeres el $59,7 \%$, y a los varones, el $40,3 \%$.

En cuanto a la elección sexual del varón se registran, en primer lugar, con una gran variedad de formas, unidades del campo de la esfera de significación de otro de los conjuntos estratégicos del dispositivo de la sexualidad, la homosexualidad, junto a otras alternativas de menor frecuencia, como el travestismo, la transexualidad y la bisexualidad: puto, gay, trolo, putazo, homosexual, maricón, maraca, chupa picho, travesti, trabuco, traga todo, te la comés, troglodita, culeado, marica, traga sable, raro, petero, travesaño, trolín, trolazo, cola rota, te gusta el trozo, afeminado, follado, chupa verga, trabapajero, putazo, chupa pija, flogay, flogger puto, floggey puto, bisexual, transexual, histérico, traga leche, qué es lo que te hacés el macho, poco hombre, te la comés, que es lo que cruzás las piernas como esas minas que te abrieron el culo y lo querés cerrar.

Como puede advertirse, se insulta, principalmente, la elección del hombre que no sigue las pautas heteronormativas. La importancia de este conjunto se basa en la cantidad de vocablos y expresiones registradas (38), en las que se sancionan conductas que se desvían del sexo heterosexual lícito. En este mismo sentido, nos parece interesante resaltar la identificación con la homosexualidad de la tribu urbana flogger. 
Para completar este primer aspecto del análisis del centro, nos detenemos en el registro, solo en informantes varones, del vocablo sidoso, vinculado con lexemas relacionados con la homosexualidad, en el sentido de que aparecen siempre en las encuestas junto a términos que la refieren. Esto nos permite afirmar que, amén de la manifestación de homofobia, la aparición de estas unidades evidencia prejuicios y desconocimiento del tema.

Siguen en importancia unidades relativas a la actitud pasiva que pudiera tener el varón con la mujer, en las que predomina la sanción al consentimiento, explícito o implícito, de la infidelidad femenina: cornudo, guampudo, guampa, bolsa de cuernos, dominado, pollerudo, conchudo. A su vez la mujer, en menor medida y con otras connotaciones, es calificada como guampuda y cornuda. El número de vocablos registrados usados para los primeros evidenciarían una concepción androcéntrica y patriarcal, de la que pareciera desprenderse que los varones son quienes están autorizados a ser infieles.

Registramos, en tercer lugar virgen, lechudo, lechoso e impotente, que degradan al varón que no tiene una actitud típica del macho heterosexual, que no se corresponde con el ideal de sujeto activo. En relación con este grupo, encontramos vocablos que vuelven a remitirnos, desde la motivación de este centro, al conjunto del dispositivo de sexualidad referido a la masturbación: pajero, pajero de mierda, pajero del orto y manuela.

No se advierten como insultos términos vinculados al órgano sexual masculino, excepto mi picho, tu pito, mi chilo, mi poronga, que no suman más de diez en los varones y una quincena en las mujeres. En menor medida, como una muestra de machismo, los varones mencionan unidades referidas al tamaño del pene: tu novia vino conmigo porque la tenés chica y gordo sin chilo. Las mujeres también las mencionan: pito corto, chicito, manicito.

En relación con la sexualidad femenina registramos unidades relacionadas con una conducta no aprobada socialmente para las mujeres: puta, trola, zorra, perra, reventada, prostituta, concha caída, gato, gata, petera, turra, yegua, atorranta, revolcada, esquinera, putita, acabada, ramera, te la comés tu concha, promiscua, sos una cualquiera, prosti, re puta, come negra putita de mierda, degradada, regalada, comida, calienta, abierta, cogida, te rompieron toda, mac donal, la más comida de todas, tenés un agujero más grande que la capa de ozono, tenés quinientos padres, sucia barata, cualquiera, lechuda, golfa, arrastrada, te comen la cajeta, te cogieron, te culean, barata, vendida, cuatro pesos, culeada, fácil, bandida, concha partida, calentona, nena fácil, olor a leche, lechosa, la loca del picho, rota, culeada, culo roto, chupa pija, calienta pija y sifilosa.

Pueden verse en estos ejemplos y en su abundancia (61) representaciones peyorativas de la mujer, en relación con prácticas socialmente desaprobadas que implican la transgresión de determinados límites. Resulta interesante destacar que sifilosa solo aparece entre mujeres, unido a vocablos que califican a la mujer como 
prostituta. Pareciera que esta enfermedad, reducida a la esfera femenina, evidenciara una sanción a libertad sexual.

Esta representación negativa de la mujer se complementa con lexemas que la describen como lesbiana, tortillera, trola, torta, torta frita, milanesa, milanga, maricona. Desde el punto de vista de la motivación de este centro, la manifestación de estos insultos, mencionados casi exclusivamente por mujeres, nos permite suponer que son ellas las que sancionan la homosexualidad femenina, y en mucha menor medida, los varones.

Mención especial merecen vocablos que aluden a otro de los conjuntos estratégicos del dispositivo de la sexualidad, la mujer histérica: hacete coger, andá a coger, frígida, mal cogida y mal comida.

Este conjunto de lexemas, referidos al universo femenino, marcan dos representaciones sociales de la mujer: la prostituta y la histérica. En lo que respecta a este último se alude a un "cuerpo íntegramente saturado de sexualidad", cuerpo que "fue integrado, bajo los efectos de una patología que le sería intrínseca, al campo de las prácticas médicas; y por último fue puesto en comunicación orgánica con el cuerpo social" (Foucault, 2011, p. 100).

Se mencionan, además, términos referidos a las partes del cuerpo de la mujer en los que prevalecen los disfemismos concha y cajeta, en un amplio abanico de combinaciones: la concha de tu madre, la cajeta, conchuda, la concha de la lora, cajetuda, la concha de tu hermana, la cajeta de tu hermana, la concha, la concha de tu mamá, conchudo de mierda, la concha que te parió, la cajeta de tu mamá, la cajeta de tu abuela, la reconchinchísima, la concha de mi madre, cajetudo de mierda, pero la concha, la cajeta bien de tu madre, la re cajeta de tu mamá, la cajeta de tu pendeja, la concha de tu vieja, la concha de tu abuela, concha caída, a tu mamá por el culo, tu agujero.

Encontramos, también, lexemas que hablan claramente de una visión violenta y falocrática de la sexualidad, ya que se registran 40 vocablos como chupame, sobame, chupame la pija, chupame un huevo, agarrame, chupame el picho, chupala, echate una paja, andá a pelar banana con tu culo, agárrame esta, te voy a romper la cola, metételo en el culo, te cojo todo, chupame el orto, haceme un pete, te cojo puto, chupámela, agarrame la pija, eh chúpame la verga, agarrá esta, metete un palo en el culo y dalo vuelta hasta marearte, tragá todo puto, colgate de esta, haceme clic, haceme doble clic, te rompo, te parto, agarrame el micrófono, agarrame el mause, te rompo el clítoris, a tu hermana la hago, le voy a coger a tu hermana, vení probá, te voy a romper todo, te parto en ocho, callate o te cojo, te cojo, te quiero coger, cogeme, te toco tu teta, sobame -los dieciséis últimos solo en mujeres-, y el anglicismo fack you. Como puede advertirse, la violencia de origen masculino queda plasmada no solo en la variedad de vocablos sino también en el número de términos registrados.

A través de estas formas lingüísticas es posible constatar, desde un plano verbalcomunicativo, el grado de violencia que subyace en el sustrato cognitivo de los 
jóvenes. La mujer es concebida como objeto y destinataria pasiva de la acción sexual, al servicio de una sexualidad violenta y descontrolada.

Es interesante observar que unidades como pervertido, degenerado, sátiro, leche andante, calentón, pijudo, sexópata, baboso, promiscuo, mujeriego solo se registran entre mujeres. Esto nos lleva a suponer que el deseo sexual incontrolado está permitido entre los varones y que, por consiguiente, como cualidad masculina, no es objeto de sanción.

Asimismo, observamos abundancia de insultos sexuales referidos al entorno familiar femenino: tu mamá, la puta que te parió, hijo de re mil puta, tu hermana, hijo de mil puta, la que te parió, tu madre, tu mamá en cuatro, hijo de perra, tu tía, tu vieja, hijo de tu madre, tu abuela, la puta que te re parió, la puta madre que te parió, tu mamá la trola, tu madrina, tu mamá se la come, tu mamá la más puta, a tu mamá en cuatro, tu suegra, tu yerna, tu mamá me chupa toda la noche, tu mamá en ocho, hijo de un buque lleno de putas, a tu mamá la llaman billetera, tu madre me hace unos petardos, tenés quinientos padres, tu abuelita en tanga, la conchita peluda y llena de flujo de tu madre que abre las piernas y deja meterse como a tragamonedas; y al entorno de una pareja: la cajeta de tu pendeja, tu mujer, la cola de tu mujer.

Podemos suponer que el entorno familiar constituye, desde la perspectiva masculina, un ámbito donde ejercen su poder y su dominio.

Registramos, también, dos vocablos muy significativos -come indias y come negras - solo en informantes de escuelas privadas. Ambos casos nos permitirían suponer que expresan, además de la cosificación de la mujer, el abuso de poder por parte de quienes se encuentran en una condición social de superioridad.

Encontramos, finalmente, dos insultos poco comunes en estudios de la temática, vinculados con la pederastia y el incesto, hecho que no es común en estudios sobre la temática: te come tu papá y come niños.

\section{Aportes para las representaciones de la sexualidad}

Consideramos, con Jodelet (1985, p.473), que las representaciones "constituyen modalidades de pensamiento práctico orientadas hacia la comunicación, la comprensión y el dominio del entorno social, material e ideal" y que, "en tanto tales, presentan características específicas a nivel de la organización de los contenidos, las operaciones mentales y la lógica".

Organizamos, en consecuencia, los contenidos sobre la base de la sistematización de la información -que nos proporcionan los resultados de los cuatro centros de interés-, para la elaboración de representaciones sobre la sexualidad de nuestros informantes, estudiantes secundarios de la región NEA.

En primer lugar, en el centro - temáticamente más acotado- Las partes del cuerpo, se rescatan las siguientes cuestiones: la mayor libertad de los hombres tanto en el 
registro de unidades que remiten a las partes del cuerpo relacionadas con el sexo, como en la utilización de disfemismos para referirse a la genitalidad femenina, hecho que se corrobora en la mayor frecuencia de estas unidades en los informantes varones.

En segundo lugar, en lo que respecta a Profesiones y oficios, se constata el registro de actividades relacionadas, directa o indirectamente, con la prostitución y la referencia a prácticas sexuales violentas.

En tercer lugar, en el centro Juegos y distracciones domina, en lo que respecta a la sexualidad, la referencia, directa o sugerida, a la masturbación. Observamos, además, la presencia de unidades que vuelven a referir a la violencia en las prácticas sexuales.

En cuarto lugar, abordamos el centro de interés más abierto desde el punto de vista temático. En principio, consideramos con Meccia (2006, p. 32) que la construcción selectiva de los contenidos de las representaciones sociales se materializa si se los restaura dentro de una trama cultural en la que existen relaciones de dominación, en el caso de nuestra región, la de la cultura heteronormativa. Esto explicaría la presencia, en este último centro ¿Cómo insultamos?, de referencias negativas respecto de la diversidad sexual en general y de la homosexualidad en particular, la estigmatización de determinadas enfermedades de transmisión sexual, la imagen muchas veces negativa de la mujer y las constantes referencias a la superioridad masculina heterosexual.

Podemos afirmar entonces que los centros de interés revelan, por un lado, estados de poder que posibilitan ver o hablar sobre algo en un determinado momento y lugar o a partir de una posición social estratégica, que refleja las normas derivadas de esa posición o de las ideologías relacionadas con el lugar que se ocupa; y, por otro, se imponen e influyen sobre los comportamientos. La representación sobre la sexualidad tiene, entonces, en términos de Jodelet, una incidencia sobre la vida sexual, sobre la conducta de las personas.

A manera de conclusión de este apartado, decimos que la emergencia de las representaciones de la sexualidad en estudiantes secundarios del NEA nos hace reflexionar acerca de la educación sexual, entendida esta no como un simple juego o distracción sino como

el conjunto de aprendizajes que permiten el buen desarrollo de las capacidades sexuales, su coordinación con las demás facultades y la consecución de una buena interrelación con las personas que resulten estimulantes por su condición sexuada y sexual, consiguiendo altos valores de espontaneidad y comunicación, y también respeto y estima. (Boix, 1976, p. 16)

\section{Conclusiones}

En estas observaciones finales, retomamos los interrogantes de los que partimos para describir el tema de la sexualidad: ¿de qué hablan actualmente los jóvenes del 
NEA cuando hablan de sexualidad? y ¿qué representaciones se pueden construir con ese contenido?

Para eso, los respondimos basándonos en las muestras del léxico sincrónico disponible de las ciudades de Resistencia y Corrientes, tomadas en los años 2001 y 2008, y organizadas en conjuntos estratégicos: las partes del cuerpo relacionadas con el sexo, la prostitución, la masturbación, enfermedades de transmisión sexual, el coito, diversidad sexual -homosexualidad, transexualidad y bisexualidad-y la mujer histérica. La primera pregunta, con la presentación de las unidades que refieren a la sexualidad en cuatro centros de interés: Partes del cuerpo, Profesiones y oficios, Juegos y distracciones y ¿Cómo insultamos? La segunda, la sistematización de la información que nos brindan estos resultados de la disponibilidad léxica, valiéndonos del recurso de la conceptualización de las representaciones sociales, conscientes de que este trabajo constituye un aporte para una posterior completa representación social sobre la sexualidad de los jóvenes estudiantes del NEA.

Como ya hemos mencionado, las representaciones sociales transforman aquello que no es familiar en familiar, hacen que lo invisible se torne perceptible; en este sentido, los vocablos usados por los jóvenes del NEA, para referirse a la sexualidad, ponen de manifiesto, en los centros de interés analizados, varias cuestiones relacionadas con la sexualidad, tal como lo hemos planteado en este trabajo. En Las partes del cuerpo, observamos que los informantes varones superan ampliamente a las informantes mujeres en el registro de términos referidos a partes del cuerpo que remiten al sexo, entre los que encontramos un número importante de disfemismos para nombrar la genitalidad femenina. Registro de actividades vinculadas con la prostitución y de prácticas sexuales con marcas de violencia, en Profesiones y oficios. En el centro Juegos y distracciones predomina la referencia a la masturbación y vuelven a aparecer referencias a prácticas sexuales violentas. Por último, en el centro ¿Cómo insultamos?, nos encontramos con unidades que aluden, de forma negativa, a la imagen de la mujer, a la diversidad sexual y a las enfermedades de transmisión sexual; además, dominan en el centro las unidades que señalan la superioridad masculina heterosexual.

\section{BIBLIOGRAFÍA}

Austin, J. (2003). Cómo hacer cosas con palabras. Buenos Aires: Paidós.

Boix, F. (1976). De la repressió a la psicopedagogia sexual. Barcelona: Nova Terra.

Butler, J. (2007) El género en disputa. El feminismo y la subversión de la identidad. Barcelona: Paidós.

Donni de Mirande, N. (1992) El Español Actual Hablado en la Argentina. En C. Hernández Alonso (Coord.), Historia y Presente del Español de América (pp. 383-410). Valladolid: PABECAL. 
Echeverría, M. (1987). Disponibilidad léxica en Educación Media. Resultados cuantitativos, VII Seminario de Investigación y Enseñanza de la Lingüística, 102-116.

Echeverría, M. (1991). Crecimiento de la disponibilidad léxica en estudiantes chilenos de nivel básico y medio. En H. López Morales (Ed.) La enseñanza del español como lengua materna (pp. 61-78). Río Piedras: Universidad de Puerto Rico.

Enríquez González, J. A. (1999). El aparato genital humano. Análisis sexista de su semántica, en Fernández de la Torre Madueño, M. A., Medina Guerra, A. M. y Taillefer de Haya, L. (Eds.) El sexismo en el lenguaje. Tomo II, Málaga, CEDMA, pp. 391-397.

Foucault, M. (1985). Saber y verdad. Madrid: Ediciones de La Piqueta.

Foucault, M. (2011). Historia de la sexualidad. La voluntad de saber. Buenos Aires: Siglo XXI.

Jodelet, D. (1984). La representación social: fenómeno, concepto y teoría. En Moscovici, S. (Comp.) Psicología Social II (pp. 469-494). Barcelona: Paidós.

Kleiber, G. (1990). La semántica de los prototipos. Categoría y sentido léxico. Madrid: Visor.

López Morales, H. (1999). Léxico disponible de Puerto Rico. Madrid: Arco Libros.

Lotman, I. M. (1996). La semiosfera (Tomos I y II). Madrid: Cátedra.

Martínez Garrido, E. (2005). Breves consideraciones semánticas de género en torno al léxico "amoroso", la retórica "erótica" y a las paremias misóginas. Cuadernos de Filología Italiana, vol. 12, 171-186.

Mateo García, M. V. (1996). Estratificación social del léxico de la alimentación en Motril (Granada). Almería: Universidad de Almería.

Meccia, E. (2006). La cuestión gay. Un enfoque sociológico. Buenos Aires: Gran Aldea Editores.

Moreno Fernández, F., Moreno Fernández, J. E., y García de las Heras, A. E. (1995). Cálculo de disponibilidad léxica. El programa LexiDisp. Lingüística, 7, 243-249.

Real Academia Española (2001). Diccionario de la lengua española. Madrid: Espasa Calpe.

Real Academia Española y Asociación de Academias de la Lengua Española (2005) Diccionario panhispánico de dudas. Madrid: Santillana.

Sontang, S. (2008). La Enfermedad y sus metáforas. El SIDA y sus metáforas. Barcelona: Editorial De Bolsillo.

Wingeyer, H. R. (2007). Léxico disponible de Resistencia. Departamento de Filología de la Facultad de Filosofía y Letras de la Universidad de Alcalá, Alcalá de Henares, Tesis Doctoral inédita.

Wingeyer, H. R., Gusberti, J. V. y Trevisán, O. N. (2011). Análisis del tema: partes del cuerpo en la disponibilidad léxica de Resistencia y Corrientes. Itinerarios Educativos, año 5, núm. 5. Santa Fe: Ediciones UNL. 